\title{
GLOBALIZAÇÃO E SEUS IMPACTOS NA VULNERABILIDADE E FLEXIBILIZAÇÃO DAS RELAÇÕES DO TRABALHO EM SAÚDE
}

\section{GLOBALIZATION AND ITS IMPACTS ON VULNERABILITY AND ON THE FLEXIBILIZATION OF THE LABOR RELATIONSHIPS IN HEALTH}

\author{
Solange Baraldi ${ }^{1}$ \\ Monica Yolanda Padilla Díaz ${ }^{2}$ \\ Wagner de Jesus Martins ${ }^{3}$ \\ Daniel Alvão de Carvalho Júnior ${ }^{4}$
}

\begin{abstract}
Resumo No processo de globalização, a flexibilização laboral e a vulnerabilidade da população tendem a aumentar. O objetivo deste estudo é analisar a situação do mercado de trabalho no setor saúde brasileiro à luz dos conceitos da vulnerabilidade no contexto da globalização. Foi realizado um estudo sistemático a partir da produção técnico-científica nacional e internacional. Os conceitos de vulnerabilidade foram tomados como base teórica, pois consideram não somente questões pertinentes ao setor saúde como também sua relação com a estrutura e superestrutura social, passível de integrar-se no marco das condições de trabalho desse setor, que em termos das condições de trabalho podem elucidar a formulação de políticas mais humanas aos trabalhadores.
\end{abstract}

Palavras-chave políticas de saúde; vulnerabilidade; flexibilização laboral.

\begin{abstract}
In the globalization process, labor flexibilization and the population's vulnerability tend to increase. The purpose of this study is to analyze the situation of the work market in the Brazilian health sector under the light of vulnerability in the globalization context. A systematic study was carried out based on national and international technical and scientific production. The concepts of vulnerability were used as the theoretical base, as they consider not only issues that are pertinent to the health sector, but also to its relationship with the social structure and superstructure, which can integrate with each other in the framework of this sector's work conditions, which, in turn, can elucidate the formulation of policies that are more human for the workers.
\end{abstract}

Keywords Health policies; vulnerability; labor flexibilization. 


\section{Introdução}

O termo globalização foi incorporado à linguagem comum como um indicativo de grandes possibilidades para o desenvolvimento da humanidade, tendo em vista as transformações sociais e econômicas ocorridas no processo produtivo desde os anos 60 (fluxo de comunicação, internacionalização das relações de produção, novas tecnologias etc.). Esta percepção sobre a globalização estabelece um panorama unilateral, e de certa forma idealizado, acerca das possibilidades de acesso aos diversos recursos existentes: naturais, financeiros, informacionais, relacionais, entre outros (Galvão, 2008). Este significado dado ao termo globalização objetiva apresentar o fenômeno como sendo "(...) a superação da velha compartimentação nacional por uma atuação mais livre dos indivíduos no mercado" (Chesnais, 1996, p. 34).

Um dos efeitos deste processo materializa-se no fato de "(...)hoje cada vez mais e mais pessoas estão entre as categorias da pobreza e riqueza(...)" assim sendo, "(...)os grandes globalizam, e os pequenos adaptam-se" (Beck, 1999, p. 3-4).

O fenômeno da globalização, para alguns estudiosos, não está sendo analisado sob o ponto de vista da problemática do valor, mas, sim, a partir de suas manifestações externas como comunicação, informática, transportes, formação de blocos econômicos, comércio intrafirmas, internacionalização da produção.

Todas estas características da atual fase do modo de produção capitalista resultam insuficientes para explicar sua natureza e nos questionam acerca das novas relações que ora se estabelecem na dinâmica mundial (Valencia, 2003). Mészáros, por exemplo, aponta a existência de uma taxa decrescente do valor de uso das mercadorias:

(...) essa tendência decrescente do valor de uso das mercadorias (inclusive o trabalho), ao reduzir a sua vida útil e, desse modo, acelerar o ciclo reprodutivo, tem se constituído num dos principais mecanismos graças ao qual o capital vem atingindo incomensurável crescimento ao longo da história (Mészáros, 2002, p. 567).

A partir desse cenário, este artigo objetiva analisar a situação do mercado de trabalho no setor saúde do Brasil à luz dos conceitos da vulnerabilidade no contexto da globalização.

\section{Considerações metodológicas}

Elegemos a dialética como instrumento metodológico para a compreensão da teoria, dos dados empíricos e de seus relacionamentos. O pensar dialéti- 
co pede para reconsiderar o homem no interior de determinada sociedade, contextualizando-a num específico momento histórico. Possibilita compreender a prática social, entendida como em constante transformação, onde os atores sociais e a estrutura social são capazes de modificá-la (Minayo, 1998; Demo, 2000).

A partir deste olhar, realizou-se revisão atualizada e sistemática em periódicos nacionais e internacionais sobre o tema mercado de trabalho em saúde, mantendo o diálogo com o marco teórico e metodológico descrito.

Para esta análise foram cumpridas as seguintes etapas: análise bibliográfica sobre os desdobramentos da flexibilização laboral no setor saúde desde a década de 1990; acesso a algumas bases de dados do governo brasileiro que propiciam tanto uma visão geral da tendência do mercado de trabalho brasileiro quanto uma visão particular acerca do mercado de trabalho no setor saúde, leia-se Sistema Único de Saúde (SUS), conforme o Quadro 1.

Quadro 1

\begin{tabular}{llll}
\hline \multicolumn{2}{l}{ Bases de dados governamentais pesquisadas } & & \\
\hline Tema & Fonte & Banco de dados & URL \\
\hline Mercado trabalho em geral & Instituto de & IpeaData & www.ipeadata.gov.br \\
& Pesquisa & Macroeconômico, & \\
& Econômica & temas emprego e & \\
& Aplicada (Ipea) & população & \\
& & & \multirow{2}{*}{ www.datasus.gov.br } \\
Recusos humanos no SUS & Ministério da Saúde & Indicadores e & \\
& (MS) & dados básicos (IDB) & \\
& & & \\
\end{tabular}

Nota: O autor (2008).

Estas bases foram eleitas a partir dos seguintes critérios: conter dados atualizados acerca do mercado de trabalho em geral e da saúde em particular, e outros de interesse, como demografia, por exemplo; facilidade de acesso; e confiabilidade na qualidade do dado.

\section{Conceito de vulnerabilidade}

O conceito de vulnerabilidade é fortemente alicerçado na idéia de que tanto a saúde quanto a doença são produtos do modo como o homem vive em sociedade. Portanto, ambos são determinados socialmente. Transformações no mundo do trabalho, para a 'classe-que-vive-do-trabalho' (Antunes, 2003), delimitam as formas de produção da saúde e da doença. 
Não obstante, o próprio conceito de determinação social da saúde (DSS) possui trajetória histórica própria (Buss, 2007).

A Organização Internacional do Trabalho (OIT) apresentou a terminologia 'vulnerabilidade social do trabalhador' no sentido de "um estado de elevada exposição a determinados riscos ou incertezas, combinado com uma capacidade diminuída para se proteger ou defender-se deles e para fazer frente a suas conseqüências negativas" (OIT, 2003, p. 43). É neste sentido que usamos o conceito de vulnerabilidade.

De acordo com Sánchez e Bertolozzi (2007), este conceito tem sido explorado em favor do reconhecimento de questões estruturais para o embasamento das práticas de saúde relativas aos indivíduos, grupos e, conseqüentemente, na formulação de políticas de saúde.

No que diz respeito à saúde do trabalhador, o termo vulnerabilidade poderia traduzir-se como ferramenta para elaboração de diagnósticos que possam orientar ações e processos decisórios na formulação e implementação de políticas públicas e, portanto, ao desenvolvimento de metodologias que visam à caracterização dos distintos graus de proteção social.

Neste sentido, a OIT apresentou uma tentativa de relacionar as dimensões 'tipo de relação de trabalho', 'natureza da empresa/organização contratante' e 'escolaridade' dos recursos humanos empregados. O Quadro 2 sistematiza esta proposição.

Quadro 2

\begin{tabular}{llll}
\hline \multicolumn{2}{l}{ Grau de vulnerabilidade social dos trabalhadores segundo suas condições no mercado de trabalho } \\
\hline Vulnerabilidade & Relação de trabalho & Empresa & Qualificação \\
\hline Patrão & Todas & Todos \\
Assalariado & Grande & Todos \\
Assalariado & Pública & Todos \\
Conta própria & - & Nível superior \\
Assalariado & Pequena & Todos \\
Conta própria & - & Sem nível superior \\
Sem salário ou & desocupao & Todos
\end{tabular}

Fonte: Panorama Laboral da América Latina, OIT, 2003

Por ser entendido como algo diferente do risco epidemiológico, que relaciona as formas com as quais o sujeito acessa os diferentes recursos produzidos na e através da vida em sociedade, o conceito de vulnerabilidade pode ser utilizado como um instrumento para a formulação de políticas públicas. 


\section{Aspectos relacionados à flexibilização laboral e vulnerabilidade do trabalhador}

No Brasil, em 1990, a população economicamente ativa (PEA) era constituída de um pouco mais de 49 milhões de cidadãos, com uma população ocupada de 16,8 milhões; já em 2006, passa a contar com 70,9 milhões e uma população ocupada de 20,2 milhões (Ipea, 2008). Estes dados, produzidos pelo governo brasileiro, indicam um crescimento da população desocupada: se em 1990 eram cerca de 32,2 milhões, em 2006 são 50,7 milhões os brasileiros desocupados. O fantasma do desemprego ronda a maioria da população brasileira, tornando-a propensa a aceitar variadas condições de trabalho (carga horária, tipo de vínculos, remuneração, condições ambientais).

Gráfico 1

Evolução da PEA e população ocupada no período de 1990 a 2006

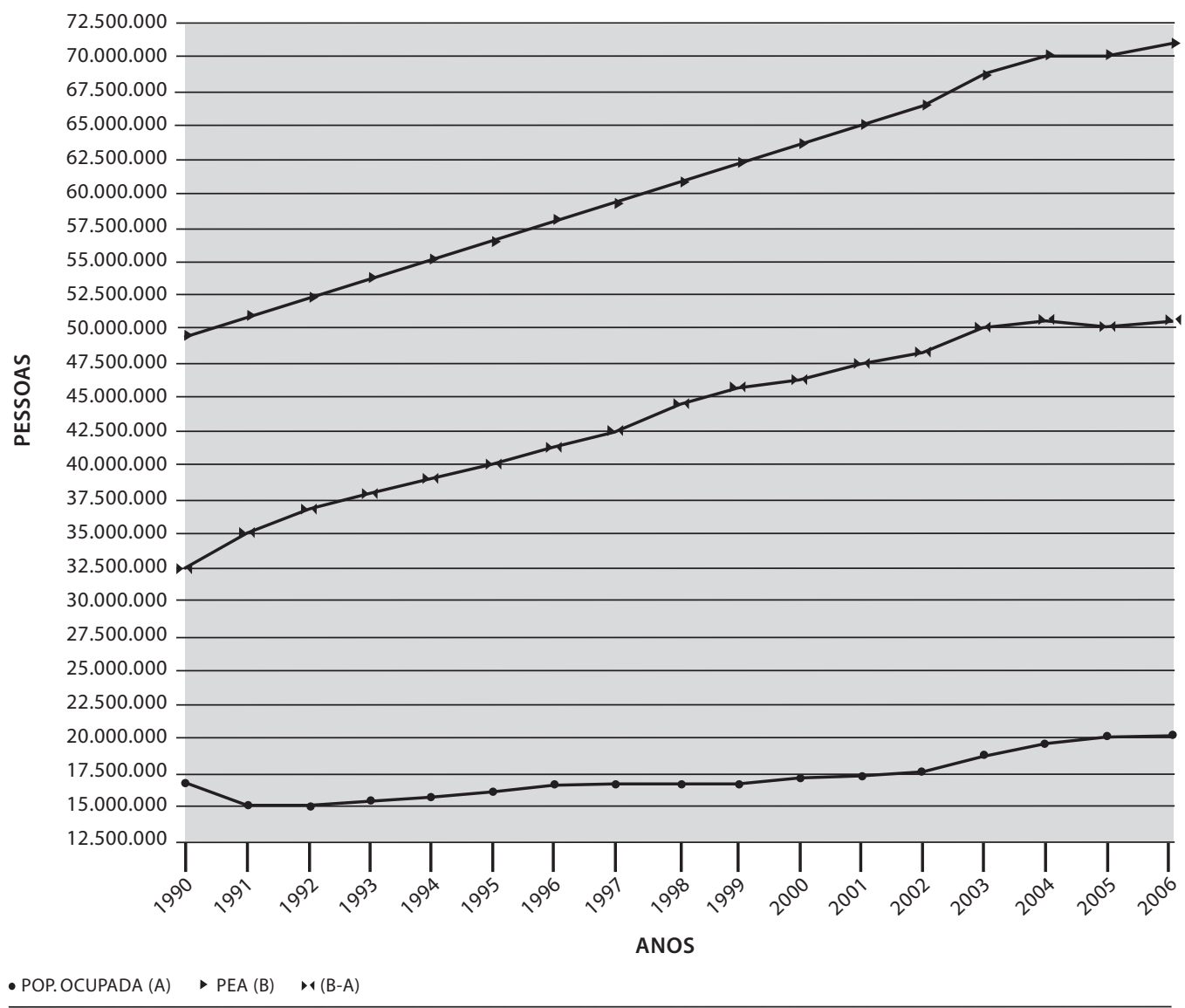


Historicamente, no Brasil, o setor saúde possui presença marcante do Estado. Em 1988, com a promulgação da Constituição federal e da criação do Sistema Único de Saúde, o Estado consolida-se como principal empregador de mão-de-obra nesta área (Lima, 2005). De acordo com o Ministério da Saúde (Brasil, 2007, p.63), do total de 1.436.987 ocupações inseridas nos serviços de saúde, 91,9\% estão vinculadas ao SUS.

O início dos anos 90 marca a hegemonia do neoliberalismo em diversos espaços da vida brasileira, inclusive na gestão pública federal. Portanto, o recém-nascido SUS, com seus princípios de universalidade de acesso aos serviços, financiamento público, entre outras características nãoliberalizantes, enfrenta um ambiente hostil para seu desenvolvimento.

Neste período, a desregulamentação das relações de trabalho favorece o aumento da quantidade de contratos de trabalho 'flexíveis', sendo os instrumentos jurídicos utilizados os mais diversos: contratação de cooperativas de profissionais, utilização de acordos de cooperação com organizações internacionais, entre outros. Cherchiglia et al. (1998) apontam que a contratação de mão-de-obra via agências empregadoras, cooperativas de trabalhadores, subcontratação de microempresas de profissionais e organizações internacionais figuram num elenco de soluções gerenciais e habituais do setor saúde, que possibilita a resolução das demandas emergentes e o enquadramento a certas regras legais, como a Lei Camata (lei complementar $\mathrm{n}^{\circ}$. 82, de 27 de março de 1995), que disciplina os limites das despesas de custeio do Poder Executivo na forma do artigo 169 da Constituição Federal.

No setor saúde, a flexibilização das relações de trabalho também tem proporcionado condições variáveis de trabalho, multiplicidade de vínculos e leque variável do gozo de direitos trabalhistas. Embora haja relatos de maior liberdade no tocante a negociações entre empregado-empregador, com respeito a salário, carga horária e direito a férias, é sabido que esta situação depende da demanda e oferta de profissionais. Por exemplo, em estudo realizado com enfermeiras que prestavam serviço (contratos temporários) para o governo federal, alguns elementos foram evidenciados no marco das relações flexíveis de trabalho: pagamento por produção; divisão fragmentada do trabalho; critérios de seleção e remuneração salarial variável; trabalho a distância; profissionais qualificados e polivalentes; sensação de autonomia e liberdade profissional; reduzida governabilidade e poder na tomada de decisão; dedicação comprometida mediante múltiplos vínculos e falta de tempo; presença marcante do que é 'novo'; programação de atividades substituindo modelos de planejamento; e maciça operacionalização sistemática das ações (Baraldi, 2005).

De acordo com o Ministério da Saúde (Brasil, 2007, p. 56), do total de 870.361 profissionais de saúde de nível superior, a proporção de empregos denominada 'parcial' varia entre 17,3\% (engenheiro clínico) a 75,0\% 
(sanitarista), destacando-se acima de 50\% para algumas profissões já conhecidas pelo múltiplo emprego, a saber: clínico geral $(62,7 \%)$, físico médico $(52,2 \%)$, fisioterapeuta $(57,4 \%)$, fonoaudiólogo $(65,1 \%)$, geriatra $(57,1 \%)$, gineco-obstetra $(56,8 \%)$, nutricionista $(55,1 \%)$, odontólogo $(64,7 \%)$, pediatra $(64,7 \%)$, psicólogo $(66,9 \%)$, psiquiatra $(70,6 \%)$, radiologista $(52,9 \%)$, sanitarista $(75,0 \%)$, outras especialidades médicas $(56,0 \%)$. Vale notar que, das 24 profissões de saúde analisadas, 14 possuíam vínculos parciais predominantemente, indicando a possibilidade de múltiplos trabalhos. É possível inferir que tal situação pode estar relacionada com as características destas profissões, que propiciam, em sua maioria, a vinculação em mais de um emprego, pela necessidade do trabalhador em complementar a renda com outros vínculos empregatícios.

Considerando que, no Brasil, o principal empregador de pessoas no setor saúde é o Estado (municípios, estados e União), a operacionalização do processo de 'flexibilização' dá-se em um duplo movimento: a) ausência de concursos públicos - em obediência à Lei Camata; e b) utilização intensiva de contratação de pessoas através de organismos internacionais, organizações não governamentais ( $\mathrm{ONG}$ 's), organização da sociedade civil de interesse público (Oscip's), cooperativas, entre outras organizações.

Em razão de o SUS ser constituído por princípios que prezam pela qualidade da assistência prestada, especialmente nos quesitos acessibilidade e integralidade, a oferta de boas condições de trabalho aos profissionais de saúde é fundamental para a materialização desses princípios. Desta forma, o conceito de vulnerabilidade pode ser utilizado como um parâmetro que abarca componentes de caráter multifatorial. Em termos das relações de trabalho pode contribuir para a identificação de políticas públicas que tornam mais ou menos vulneráveis estes profissionais, que já trabalham em ambientes majoritariamente hostis e insalubres, os quais denominamos de políticas pró ou antivulnerabilização, conforme sugere o Quadro 3.

Quadro 3

\begin{tabular}{ll}
\hline Políticas de pró-vulnerabilização e antivulnerabilização do trabalhador no atual quadro global, 2006 \\
\hline Pró-vulnerabilização & Antivulnerabilização \\
\hline Acessibilidade aos serviços de saúde prejudicada & Garantia da acessibilidade aos serviços de saúde \\
Mercado de trabalho e formação flexibilizados & $\begin{array}{l}\text { Eqüidade no tratamento das profissões e } \\
\text { ocupações, promovendo-lhes grau satisfatório de } \\
\text { autonomia e sentido do trabalho }\end{array}$
\end{tabular}

Desorganização das categorias profissionais na Sindicalização luta por seus direitos 
Políticas de pró-vulnerabilização e antivulnerabilização do trabalhador no atual quadro global, 2006

\begin{tabular}{|c|c|}
\hline Pró-vulnerabilização & Antivulnerabilização \\
\hline $\begin{array}{l}\text { Precarização das relações de trabalho: cobertura } \\
\text { parcial ou sem cobertura de proteção social; } \\
\text { múltiplo emprego; extensão da jornada de } \\
\text { trabalho em mais de } 40 \text { horas semanais }\end{array}$ & $\begin{array}{l}\text { Contrato de qualidade (carga horária, benefícios e } \\
\text { proteção social garantida), buscando o equilíbrio } \\
\text { possível entre empregador-trabalhador, } \\
\text { esimulando vínculos full time. } \\
\text { Proposições diferenciadas atendendo às } \\
\text { especificidades regionais }\end{array}$ \\
\hline Reduzido nível de competência pofissional & $\begin{array}{l}\text { Sólidas políticas de educação continuada e } \\
\text { desenvolvimento profissional. } \\
\text { Proposições diferenciadas atendendo às } \\
\text { especificidades regionais }\end{array}$ \\
\hline $\begin{array}{l}\text { Ajustes em benefício exclusivo da eficiência e } \\
\text { custo-benefício }\end{array}$ & $\begin{array}{l}\text { Fortalecimento de instâncias representativas nas } \\
\text { corporações, de modo a garantir interesses do } \\
\text { trabalhador. } \\
\text { Fortalecimento da capacidade de gestão } \\
\text { descentralizada. } \\
\text { Proposições diferenciadas atendendo às } \\
\text { especificidades regionais }\end{array}$ \\
\hline Processo de trabalho fragmentado & $\begin{array}{l}\text { Compreensão do sentido do trabalho na linha de } \\
\text { produção. } \\
\text { Proposições diferenciadas atendendo às } \\
\text { especificidades regionais }\end{array}$ \\
\hline $\begin{array}{l}\text { Fragmentação das equipes acirrando a divisão } \\
\text { social do trabalho }\end{array}$ & $\begin{array}{l}\text { Construção contínua do trabalho em equipe e } \\
\text { processo de avaliação heterogêneo }\end{array}$ \\
\hline
\end{tabular}

Fonte: $\mathrm{O}$ autor, 2008.

\section{Conclusão}

O processo de mundialização do capital (globalização) exige que a relação entre o empregador e o empregado seja 'flexível'. Flexível do ponto de vista do empregador. Desta forma, os 'rígidos' regramentos jurídicos que protegiam o trabalhador vêm sendo substituídos, reformados, quando não eliminados.

É árdua a tarefa de formular proposições que impliquem real melhoria da condição do trabalhador, considerando-se que os modelos flexíveis e precários de relação de trabalho ainda conformam uma realidade sustentada por reformas no Estado brasileiro, o que cria as condições propícias para que se tornem paulatinamente legitimadas diante dos índices de desemprego. No tocante aos trabalhadores de saúde, a preocupação focal deve 
estar direcionada para as relações e condições de trabalho em si, vez que impactam na qualidade do serviço prestado.

Nos últimos três anos, o Ministério da Saúde e demais órgãos gestores do SUS têm incorporado em suas políticas alguns marcos que visam a minimizar a vulnerabilidade dos trabalhadores diante de situações flexíveis ou mesmo precárias de trabalho, o que pode ser percebido na realização de concursos públicos nas instâncias municipais, estaduais e federal, talvez indicando uma reversão de tendências no que foi apontado neste estudo.

\section{Notas}

1 Professora adjunta da Universidade de Brasília (UnB). Doutora em Saúde do Adulto pela Escola de Enfermagem da Universidade de São Paulo (EE/USP). <solbaraldi@unb.br> Correspondência: Universidade de Brasília, Campus Universitário Darcy Ribeiro, Faculdade de Ciências da Saúde, Asa Norte, Brasília, Distrito Federal, CEP 70904-970, Caixa Postal 4381.

2 Consultora internacional para o desenvolvimento de recursos humanos em saúde da Sub-Região Andina, da Organização Pan-Americana da Saúde/Organização Mundial da Saúde (Opas/OMS). Mestre em Investigação e Administração em Saúde pela Universidade Central do Equador. <mpadilla@per.ops-oms.org>

3 Diretor de Programas, Planejamento e Gestão da Secretaria de Ciência, Tecnologia e Insumos Estratégicos do Ministério da Saúde (SCTIE/MS). Mestre em Saúde Pública pela Escola Nacional de Saúde Pública Sergio Arouca, da Fundação Oswaldo Cruz (Ensp/Fiocruz).<wagner.martins@saude.gov.br>

${ }^{4}$ Assessor técnico do Ministério da Saúde. Mestrando em Ciências da Saúde pela Universidade de Brasília (UnB). < daniel.junior@saude.gov.br>

\section{Referências}

ANTUNES, Ricardo. Os sentidos do trabalho. São Paulo: Boitempo Editorial, 2003.

BARALDI, Solange. Supervisão, flexibilização e desregulamentação no mercado de trabalho: antigos modos, novas incertezas nos vínculos de trabalho da enfermagem. Tese de Doutorado. Escola de Enfermagem da USP, São Paulo, 2005.

BECK, Ulrich. The brave new world of work. Cambridge: Polity Press, 1999. Beck U. The brave new world of work. Cambridge: Polity Press, 1999. 
BENAVIDES, Fernando G. et al. Associations between temporary employment and occupational injury: what are the mechanisms?. Occupational and Environmental Medicine, Londres, n. 63, 2006, p. 416-421.

BRASIL, Ministério da Saúde. Secretaria de Gestão do Trabalho e da Educação na Saúde. Indicadores de gestão do trabalho e da educação no SUS. Brasília: Ministério da Saúde, 2007.

BUSS, Paulo Marchiori; PELLEGRINI FILHO, Alberto. A saúde e seus determinantes sociais. Physis, Rio de Janeiro, v. 17, n. 1, 2007. Disponível em: www.scielo.br/ scielo.php?script=sci_arttext\&pid=S0103$73312007000100006 \& \operatorname{lng}=\mathrm{en} \& \mathrm{nrm}=\mathrm{iso}$. Acesso em: 12 abr. 2008.

CHERCHIGLIA, Mariangela L. et al. Remuneración y productividad: el caso de la Fundación Hospitalaria del Estado de Minas Gerais, Brasil, 1992-1995. Revista Panamericana de Salud Pública, Washington, v. 4, n. 2, ago. 1998.

CHESNAIS, François. A mundialização do capital. São Paulo: Xamã, 1996.

DEMO, Pedro. Metodologia do conhecimento científico. São Paulo: Atlas; 2000.

GALVÃO, Olímpio J. de Arroxelas. Globalização e mudanças na configuração espacial: da economia mundial: uma visão panorâmica das últimas décadas. Revista de Economia Contemporânea [online]. 2007, vol. 11, n. 1 Disponível em: $<$ www.scielo.br/scielo.php?script=sci_abst
ract\&pid=S1415-98482007000100003\&lng $=$ pt\&nrm=iso $>$ Acesso em: 21 jul. 2008.

IPEA (Instituto de Pesquisa Econômica Aplicada). Base de dados. Mercado de Trabalho. IPEA. Brasília, 2008. Disponível em: $<$ www.ipeadata.gov.br $>$ Acesso em: 15 mar. 2008.

LIMA, Nísia Trindade et al. (Orgs.). Saúde e democracia: história e perspectivas do SUS. Rio de Janeiro: Editora Fiocruz, 2005.

MÉSZÁROS, István. Para além do capital. São Paulo: Boitempo, 2002.

MINAYO, Maria Cecília de S. O desafio do conhecimento: pesquisa qualitativa em saúde. 5. ed. São Paulo, 1998.

OIT (Organização Internacional do Trabalho). Panorama laboral 2003: América Latina y El Caribe. Lima: Oficina Regional para a América Latina y El Caribe, 2003. 124p.

SÁNCHEZ, Alba Idaly M.; BERTOLOZZI, Maria Rita. Pode o conceito de vulnerabilidade apoiar a construção do conhecimento em Saúde Coletiva? Ciência \& Saúde Coleti$v a$, v. 12, n. 2, p. 319-324.

VALENCIA, Adrián Sotelo. La reestructuración del mundo del trabajo, superexplotación y nuevos paradigmas de la organización del trabajo. México: Efraín Herrera, 2003.

Recebido em 10/04/2008

Aprovado em 28/09/2008 















